\title{
Hypoglykämien mindern die Therapietreue
}

\author{
Leichte Unterzuckerungen sind bei Typ-2-Diabetikern häufig \\ und ein Grund für schlechte Therapietreue. Die Patienten ris- \\ kieren dadurch vermeidbare Langzeitkomplikationen.
}

Diese Erkenntnisse resultieren aus der GAPP2-Studie, einer globalen OnlineBefragung von ca. 3000 Diabetikern und 1600 Diabetologen. Über die Ergebnisse berichtete Dr. Martin Grundner, ein in Frankfurt niedergelassener Diabetologe.

$80 \%$ der Patienten gaben an, schon einmal Unterzuckerungen gehabt zu haben, bei $36 \%$ war dies im letzten Monat der Fall. Nahezu die Hälfte der Patienten berichtete, schon einmal eine BasalinsulinDosis ausgelassen zu haben, in dem meisten Fällen mit Absicht. Viele Patienten spritzen Insulin auch zeitversetzt oder verringern ihre Insulindosis. Häufigstes Motiv für diese Dosisunregelmäßigkeiten war die Reduktion des Hypoglykä-
mie-Risikos. Viele Patienten gaben auch an, dass es sie belaste, das Insulin immer zur gleichen Zeit injizieren zu müssen.

Auffällig waren die Ergebnisse aus Deutschland: Hier war generell die Angst vor Hypoglykämien ausgeprägt, besonders vor Unterzuckerungen im Schlaf oder beim Autofahren. Grundners Rat: Der Arzt sollte Patienten aktiv auf Hypoglykämien ansprechen.

Schwere Hypoglykämien sind einer der häufigsten Gründe für Kliniknotaufnahmen. Sie erhöhen die Sterblichkeit sowie die Risiken für Demenz und Herzinfarkt, berichtete Prof. Andreas Liebl vom Diabetes- und Stoffwechselzentrum Bad Heilbrunn. Moderne Insuline können laut Liebl dazu beitragen, Hypoglykämien zu vermeiden: „Detemir ist das Langzeitinsulin mit der geringsten Wirkungsschwankungsbreite von Tag zu Tag, einem wichtigen Risikofaktor für Hypoglykämien. Zudem führen Hypoglykämien bei Detemir-Behandlung früh zu Beschwerden wie Schwitzen, sodass der Patient frühzeitig gewarnt wird“.

Kürzlich erhielt Degludec (Tresiba $\left.{ }^{\circledR}\right)$ die europäische Zulassung, ein neues, besonders lange wirksames Basalinsulin. Die Halbwertszeit ist mit 24 Stunden doppelt so lang wie das der etablierten Basalinsuline. Daraus resultiert eine besonders gleichmäßige Wirkung, das Risiko für nächtliche Hypoglykämien ist um 26\% niedriger als bei Insulinglargin, so Liebl. Der Diabetologe hält Degludec für eine wichtige Bereicherung, die v.a. für Patienten mit Hypoglykämien und solche geeignet ist, die unzuverlässig spritzen.

Dr. med. Dirk Einecke

Quelle: Grünwalder Gespräche am 6.2.2013 in München-Grünwald, Veranstalter: Novo Nordisk

\section{Diabetes Typ 2}

\section{Neues Wirkprinzip ergänzt die Therapie}

\author{
Die Hemmung des Sodium-Glucose linked Transporter 2 (SGLT2) ist ein \\ neuer Ansatz in der oralen Diabetestherapie. Daher ist der entsprechende \\ Wirkstoff Dapagliflozin, der seit kurzem in Deutschland verfügbar ist, \\ auch mit allen bisherigen Antidiabetika gut kombinierbar.
}

der renalen Glukose-Rückresorption. „Mit Dapagliflozin wird dieser Mechanismus gehemmt und die überschüssige Glukose mit dem Urin ausgeschieden", sagte Prof. Roland E. Schmieder aus Erlangen. Dass dieser Wirkansatz effizient ist, belegen die Daten von elf kontrollierten Phase-III-Studien, in denen die Wirksamkeit und Sicherheit von Dapagliflozin bei Typ-2-Diabetes geprüft wurde: In Kombination mit Metformin senkte das Flozin (1x tägl. $10 \mathrm{mg}$ ) innerhalb von 24 Wochen das $\mathrm{HbA}_{1 \mathrm{c}}$ im Mittel um 0,84 Prozentpunkte (vs. $-0,30 \%$-Punkte mit Metformin plus Placebo, $\mathrm{p}<0,0001$ ), was über zwei Jahre erhalten blieb. Als positive Zusatzeffekte zeigten sich moderate Gewichts- und Blutdrucksenkungen. Die Gesamthäufigkeit unerwünschter Er-

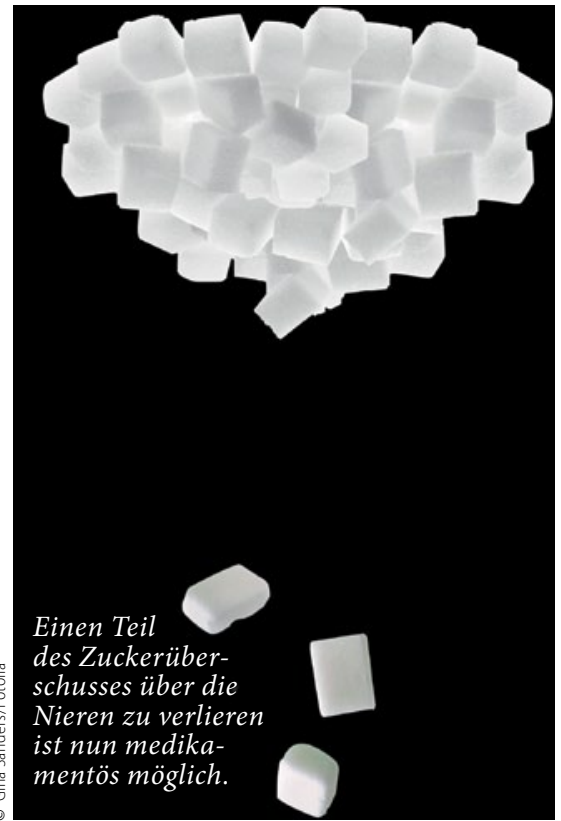

eignisse waren in den Placebo- und Verumgruppen ähnlich. In Kombination mit Metformin bestand keine erhöhte Hypoglykämietendenz.

Dr. Ellen Jahn

Pressegespräch am 24.1.2013 in Frankfurt am Main, Veranstalter: Bristol-Myers Squibb und AstraZeneca 